\title{
Spin-Vibronic Superexchange in Mott-Hubbard Fullerides
}

\author{
L. F. Chibotaru \\ Department of Chemistry, University of Leuven, Celestijnenlaan 200F, B-3001 Leuven, Belgium
}

(Received 21 December 2004; published 10 May 2005)

\begin{abstract}
In the Mott-Hubbard cubic fulleride $\mathrm{Li}_{3}\left(\mathrm{NH}_{3}\right)_{6} \mathrm{C}_{60}$ the superexchange energy is found to be much smaller than the rotational quantum for Jahn-Teller deformations at fullerene sites. This gives rise to a new type of superexchange interaction involving threefold degenerate vibronic ground states of $\mathrm{C}_{60}^{3-}$ ions. In contrast with spin-orbital models, the spin-vibronic superexchange can be only antiferromagnetic and shows a significant vibronic reduction of the superexchange amplitude, in agreement with magnetic susceptibility data. As a function of the transfer parameters, two quadrupolar fully dynamical vibronic orders with quenched vibronic moments on sites develop in the ground state.
\end{abstract}

PACS numbers: 71.20.Tx, 71.70.- d, 71.27.+a

Alkali doped fullerides have been the object of intensive investigations in the last decade [1,2]. In these crystals the electrons from alkali atoms are transferred into threefold degenerate orbitals of the fullerene sites. In the MottHubbard insulating regime, the relatively strong intrasite electron repulsion together with orbital degeneracy results in a concomitant presence of spin, orbital, and lattice (Jahn-Teller) degrees of freedom in the low-energy states. In this respect alkali doped fullerides are similar to other strongly correlated materials with orbital degeneracy [3,4]. Recently, the first cubic Mott-Hubbard fulleride, $\mathrm{Li}_{3}\left(\mathrm{NH}_{3}\right)_{6} \mathrm{C}_{60}$, has been obtained and investigated [5]. In contrast to other ammoniated fullerides [6], in this compound each fullerene site is in a crystal field of cubic symmetry preserving the threefold degeneracy of the lowest unoccupied molecular orbitals (LUMO). The magnetic susceptibilty was found to be in full agreement with an isotropic $S=1 / 2$ Heisenberg model for a bcc lattice [5]. This is neither consistent with a situation of undistorted fullerenes, where a Hund state $(S=3 / 2)$ is realized at each site, nor is it the case of static Jahn-Teller distortions as evidenced by structural data [5]. There is therefore a strong indication that the dynamical Jahn-Teller effect plays an important role in the low-energy phase of this compound.

In this Letter we address the origin of the ground state and low-lying excitations in $\mathrm{Li}_{3}\left(\mathrm{NH}_{3}\right)_{6} \mathrm{C}_{60}$. It follows that the intermediate vibronic coupling and the local cubic symmetry at fullerene sites ensures that vibronic dynamics of individual $\mathrm{C}_{60}^{3-}$ anions is not quenched. The localized spins reside in threefold degenerate vibronic ground states of fullerenes, thus giving rise to spin-vibronic superexchange between fullerene sites. Such a manifestation of the Jahn-Teller effect, which is essentially dynamic in the present case, is due to the molecular nature of the crystal and does not occur in insulating perovskites with orbitally degenerate sites, where the Jahn-Teller effect is either quenched or static $[7,8]$.

The partly occupied orbitals $t_{1 u} \gamma, \gamma=x, y, z$ at each fullerene site, interact with fivefold degenerate $H_{g}$ vibra- tions of the corresponding fullerene giving rise to the vibronic $t \otimes H$ problem $[9,10]$. In the case of one or two electrons, the $t \otimes H$ interaction splits the $t_{1 u}$ shell into the ground nondegenerate and the excited twofold degenerate levels. The minima on the ground energy surface form a twofold continuum (two-dimensional trough) $[9,10]$. The third added electron occupies the degenerate orbitals, which split as well in virtue of the Jahn-Teller theorem. The resulting adiabatic potential has a three-dimensional trough of minima in the ground state [11]. Actually there are eight modes of $H_{g}$ vibrations in $\mathrm{C}_{60}, \omega_{\mu H}=$ $271-1575 \mathrm{~cm}^{-1}$. The low-lying vibronic states can be conveniently described by an effective one-mode problem with the frequency $\bar{\omega}_{H}=\sum_{\mu} p_{\mu} / \sum_{\mu}\left(p_{\mu} / \omega_{\mu H}\right)$, where $p_{\mu}$ is the percent contribution of the $\mu$ th mode to the energy of Jahn-Teller stabilization $\left(E_{\mathrm{JT}}\right)$ [12]. Using for $p_{\mu}$ the data extracted from photoemission of $\mathrm{C}_{60}^{-}$[1] we obtain $\hbar \bar{\omega}_{H}=600 \mathrm{~cm}^{-1}$. With the current estimation of the Jahn-Teller energy for $\mathrm{C}_{60}^{3-}, E_{\mathrm{JT}}=0.15 \mathrm{eV}$, we have the ratio $E_{\mathrm{JT}} / \hbar \bar{\omega}_{H}=2$.

The electron interaction in the $t_{1 u}$ shell is described by the intraorbital $\left(U_{\|}\right)$and interorbital $\left(U_{\perp}\right)$ repulsion and the Hund rule coupling $(J)$, related by $U_{\|}-U_{\perp}=2 J$. For pristine fullerene crystals $U_{\|} \approx 1-1.5 \mathrm{eV}$ and $J \approx$ $0.03-0.05 \mathrm{eV}$ [1]. In doped crystals $U_{\|}$is further reduced by the effects of screening. In $\mathrm{C}_{60}^{3-}$ there is a competition between the Hund rule coupling, which tends to align the three electrons ferromagnetically [Fig. 1(a)], and the JahnTeller effect which tends to populate consecutively the nondegenerate orbital levels [Fig. 1(b)]. To compare the a)

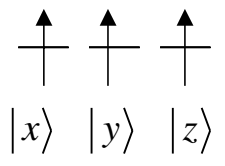

b)

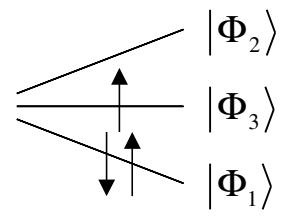

FIG. 1. Hund's (a) and Jahn-Teller (b) electronic configurations of $\mathrm{C}_{60}^{3-} \cdot\left|\Phi_{i}\right\rangle$ are adiabatic orbitals. 
energies of these two electronic configurations, we should take also into account the change of vibrational energy in the Jahn-Teller configuration [10]. Thus we have $3 U_{\|}-$ $9 J+5 / 2 \hbar \bar{\omega}_{H}$ for the Hund configuration and $3 U_{\|}-4 J-$ $E_{\mathrm{JT}}+\hbar \bar{\omega}_{H}$ for the Jahn-Teller configuration. The gain of $3 / 2 \hbar \bar{\omega}_{H}$ in the latter case is the consequence of a threedimensional trough on the lowest energy surface of $\mathrm{C}_{60}^{3-}$ [11]. Using the above estimations we conclude that the Jahn-Teller configuration is the lowest and the ground state spin is $S=1 / 2$, which is indeed found in Mott-Hubbard fullerides $[5,6,11,12]$.

The three-dimensional trough on the lowest energy surface of $\mathrm{C}_{60}^{3-}$ leads to a complex nuclear dynamics, involving free rotations of Jahn-Teller deformations along three angular coordinates and harmonic vibrations along another degrees of freedom. The solutions for low-lying states in the strong coupling approximation are $[11,12]$ :

$$
\Psi^{\mathrm{ad}}(r, \gamma, \vartheta, \varphi) \Psi_{n_{1}, n_{2}}^{\mathrm{vib}}(q, \alpha) \Psi_{L, M, K}^{\mathrm{rot}}(\gamma, \vartheta, \varphi),
$$

where $r$ denotes electronic coordinates, $q$ is the amplitude of the $H_{g}$ vibrations, and $\gamma, \vartheta, \varphi$ are three Euler angles specifying the rotation in the trough. $\Psi^{\text {ad }}$ is the adiabatic electronic function, describing the electronic state at given Euler coordinates [a Slater determinant of adiabatic orbitals, Fig. 1(b)]; $\Psi^{\text {vib }}$ describes two-dimensional vibrations across the trough; $\Psi_{L, M, K}^{\mathrm{rot}}$ is the rotational part, coinciding with the solution for a symmetric top with $K$ even. The ground state corresponds to $L=1, K=0$,

$$
\Psi_{1, M, 0}^{\mathrm{rot}}=\sqrt{\frac{2}{\pi}} Y_{1 M}(\vartheta, \varphi)
$$

and is therefore threefold degenerate. The nature of the ground state does not change when going beyond a strong coupling approximation and when multiplet effects are taken into account $[11,12]$.

The lowest vibronic states in Eq. (1) correspond to excitations in the rotational sector at $n_{1}=n_{2}=0$. The rotational quantum can be estimated as $\hbar^{2} / 2 q_{0}^{2}=\frac{1}{4} \times$ $\left(\hbar \bar{\omega}_{H}\right)^{2} / E_{\mathrm{JT}} \approx \frac{1}{8} \hbar \bar{\omega}_{H} \approx 75 \mathrm{~cm}^{-1}$, where $q_{0}$ is the radius of the trough. The solutions (1) and (2) correspond to full delocalization of Jahn-Teller deformations in the trough, i.e., to the absence of static $H_{g}$ deformations. In a crystal they can be only localized (and the adiabatic orbitals fixed at some $\gamma_{0}, \vartheta_{0}, \varphi_{0}$ ) when the crystalline interactions mixing different rotational levels exceed the spacing between them. In $\mathrm{Li}_{3}\left(\mathrm{NH}_{3}\right)_{6} \mathrm{C}_{60}$ the local crystal field at fullerene sites is of cubic symmetry, and therefore the only source of localization of Jahn-Teller deformations could be the superexchange interaction between neighboring fullerenes and the vibrational intersite interaction (phonon dispersion). To estimate the superexchange amplitude [13], we take $t \approx W /(2 z)$, where $W=0.3 \mathrm{eV}$ is the LUMO bandwidth [5] and $z=8$ for a bcc lattice, and $U=1 \mathrm{eV}$ and we obtain $4 t^{2} / U \approx 11 \mathrm{~cm}^{-1}$, which is much smaller than the estimated rotational quantum. On the other hand, the fre- quency of interfullerene optical vibrations was found $\approx$ $40 \mathrm{~cm}^{-1}$ [1] and should be even smaller in ammoniated crystals, which again does not exceed the rotational quantum. We may conclude therefore that in $\mathrm{Li}_{3}\left(\mathrm{NH}_{3}\right)_{6} \mathrm{C}_{60}$ the fullerene anions will be in threefold degenerate vibronic ground states (2).

The superexchange Hamiltonian is obtained from the following general expression [14]:

$$
\begin{aligned}
\mathcal{H}_{\text {eff }}= & \mathcal{H}_{0}+\mathcal{H}^{\prime}+\frac{1}{U} \mathcal{H}_{t} \mathcal{H}_{t} \\
& -\frac{1}{2 U^{2}}\left[\left[\mathcal{H}^{\prime}, \mathcal{H}_{t}\right], \mathcal{H}_{t}\right]
\end{aligned}
$$

where $\mathcal{H}_{0}$ contains the interactions depending on the total occupation number, with an effective $U$, and $\mathcal{H}^{\prime}$ includes the remaining intrafullerene interactions. $\mathcal{H}_{t}$ is the transfer Hamiltonian between the LUMO orbitals of the neighbor fullerene sites. In a bcc lattice all diagonal $\left(t_{\gamma \gamma}\right)$ and all off diagonal $\left(t_{\gamma \gamma^{\prime}}\right)$ transfer parameters are equal $(\gamma=$ $x, y, z)$. The third term in (3) is the Anderson antiferromagnetic contribution and the last term describes the effect of multiplet and Jahn-Teller splitting of $t_{1 u}$ shells on superexchange. Next one should average $\mathcal{H}_{\text {eff }}$ over the vibronic ground states (1) and (2) of fullerene sites. This is done in two steps. First, we average over the electronic coordinates and obtain for each pair $(i j)$ of nearest neighbor sites an operator of Heisenberg type

$$
\mathcal{H}_{i j}=K_{i j}+\left(\mathbf{S}_{i} \mathbf{S}_{j}+\frac{1}{4}\right) J_{i j}
$$

with $K_{i j}$ and $J_{i j}$ still dependent on the Euler angles of two fullerenes. These parameters are further averaged over wave functions (2) and become $9 \times 9$ matrices in the space of vibronic states of the two sites. The resulting Hamiltonian describes the spin-vibronic exchange interaction, which formally resembles the spin-orbital superexchange $[7,8]$ but acts in the space of vibronic rather than orbital states.

Given the threefold symmetry of nearest neighbor pairs, the intersite electron transfer is only nonzero between the $t_{1 u}$ orbitals with the same projection of the orbital momentum $(-1,0,1)$ on the axis of a given pair: $t_{0}=t_{x x}+2 t_{x y}$, $t_{1}=t_{-1}=t_{x x}-t_{x y}$. Similarly, the spin-vibronic superexchange will conserve the projection of the total vibronic momentum $M_{i}+M_{j}$ on the axis of the pair. It is convenient therefore to choose the quantization axis along the axis of the pair. Then keeping the main part of superexchange interaction (the terms arising from $\mathcal{H}^{\prime}$ do not change qualitatively the results as shown elsewhere [14]) we obtain (in units of $4 t_{0}^{2} / 50 U$ ) 


$$
\begin{aligned}
J_{i j} & =\left(8+9 \eta^{2}\right)+\left(3 \eta^{2}-4\right)\left(n_{0}^{a}+n_{0}^{b}\right)+\left(2+\eta^{2}\right) n_{0}^{a} n_{0}^{b} \\
& +\frac{1}{\pi}\left[\eta^{2} a_{1}^{\dagger} a_{-1} b_{-1}^{\dagger} b_{1}\right. \\
& \left.+\frac{\eta}{2}\left(a_{1}^{\dagger} a_{0}-a_{0}^{\dagger} a_{-1}\right)\left(b_{0}^{\dagger} b_{1}-b_{-1}^{\dagger} b_{0}\right)+\text { H.c. }\right], \\
K_{i j} & =\left(-9+2 \eta^{2}\right) n_{0}^{a} n_{0}^{b}+\frac{1}{\pi}\left[2 \eta^{2} a_{1}^{\dagger} a_{-1} b_{-1}^{\dagger} b_{1}\right. \\
& \left.+\eta\left(a_{1}^{\dagger} a_{0}-a_{0}^{\dagger} a_{-1}\right)\left(b_{0}^{\dagger} b_{1}-b_{-1}^{\dagger} b_{0}\right)+\text { H.c. }\right],
\end{aligned}
$$

where $a_{M}$ and $b_{M}$ are bosonic operators (analogs of orbitons in spin-orbital models [15]) corresponding to vibronic states (2) of the first and second fullerene site, respectively, constrained by the conditions $n_{0}^{a}+n_{1}^{a}+n_{-1}^{a}=1$ and $n_{0}^{b}+n_{1}^{b}+n_{-1}^{b}=1 ; \eta=t_{1} / t_{0}$ and the constant term in the expression for $K_{i j}$ was omitted.

The constant term in $J_{i j}$ is dominant for any $\eta$, which means that the coupling between nearest neighbor spins is always antiferromagnetic, in full agreement with magnetic susceptibility data for $\mathrm{Li}_{3}\left(\mathrm{NH}_{3}\right)_{6} \mathrm{C}_{60}$ [5]. In the ordered phase, the spin moments of the two antiferromagnetic sublattices will be almost unreduced with respect to the corresponding Heisenberg model, given the relative smallness of vibronic terms in $J_{i j}$. This behavior differs much from the superexchange between orbitally degenerate sites $\left(t_{2 g}^{1}-t_{2 g}^{1}\right.$ pairs) both in perovskites (titanates) $[15,16]$ and bcc lattices [14]. In the last case the interaction between spins is strongly dependent on orbital populations and can be equally ferromagnetic and antiferromagnetic, with spin moments strongly reduced by orbital fluctuations in ordered phases. The specific of spin-vibronic superexchange comes from the delocalization of the adiabatic orbitals over all three $t_{1 u} \gamma$ orbitals in each vibronic state (2). As a result the electron transfer between singly occupied adiabatic orbitals of the neighbor sites $\left(\left|\Phi_{3}\right\rangle\right.$ in Fig. 1) is never zero. The other feature of (5) is the reduction of the superexchange amplitude with respect to $4 t^{2} / U$ (for $t_{0}=t_{1}$ we obtain the reduction factor 17/50). This is again the consequence of delocalization of the adiabatic orbitals $\left|\Phi_{3}\right\rangle$ in the dynamical vibronic states (2) and corresponds to a vibronic reduction of superexchange interactions. It is just an example of Ham's reduction of electronic operators in Jahn-Teller systems [10]. Such a reduction of exchange interaction is indeed observed in $\mathrm{Li}_{3}\left(\mathrm{NH}_{3}\right)_{6} \mathrm{C}_{60}$ : from the experimental Weiss constant $\theta=-15.2 \mathrm{~K}$ [5] one derives the mean-field estimate for the exchange parameter of $5.3 \mathrm{~cm}^{-1}$ which is twice smaller than the previous estimate based on the LUMO bandwidth.

Consider now the vibronic sector of superexchange interaction. As Eq. (5) shows, the term $K_{i j}$ is an operator even in the absence of the Hund rule coupling on sites. In contrast, it reduces to a constant in spin-orbital models with $t_{2 g}^{1}$ sites, when $J=0$, for both simple cubic [16] and bcc lattices [14], which means that their classical Néel state, $\left\langle\mathbf{S}_{i} \mathbf{S}_{j}\right\rangle=-1 / 4$, is infinitely degenerate in the orbital sector. Averaging (4) over the antiferromagnetic spin or- dered state we obtain the vibronic Hamiltonian

$$
\mathcal{H}_{\mathrm{vibr}}=\sum_{\langle i j\rangle}\left(K_{i j}+\xi J_{i j}\right),
$$

where the bosonic operators in each term are defined with respect to the quantization axis of the corresponding pair and $\xi=\left\langle\mathbf{S}_{i} \mathbf{S}_{j}\right\rangle_{a f}+1 / 4$. It results that the terms $n_{0}^{a} n_{0}^{b}$ in (5) give the main contribution to (6) when $\left(t_{1} / t_{0}\right)^{2}$ is not too close to $(9-2 \xi) /(2+\xi)$. Considering this condition fulfilled we further keep only these terms. Next we introduce the vibronic momentum operators $I_{x}=i\left(a_{z}^{\dagger} a_{y}-\right.$ $\left.a_{y}^{\dagger} a_{z}\right), \quad I_{y}=i\left(a_{x}^{\dagger} a_{z}-a_{z}^{\dagger} a_{x}\right), \quad$ and $I_{z}=i\left(a_{y}^{\dagger} a_{x}-a_{x}^{\dagger} a_{y}\right)$ and the quadrupole operators $T_{x}=-\left(I_{y} I_{z}+I_{z} I_{y}\right), T_{y}=$ $-\left(I_{x} I_{z}+I_{z} I_{x}\right)$, and $T_{z}=-\left(I_{x} I_{y}+I_{y} I_{x}\right)$ for each fullerene site (they are similar to the case of $t_{2 g}^{1}$ orbital degeneracy [15]). Then the vibronic Hamiltonian can be rewritten in the form

$$
\mathcal{H}_{\text {vibr }}=\frac{4}{9} C \sum_{\mathbf{i}}\left[1+\sum_{j=0}^{3}\left(\mathbf{T}^{\mathbf{i}} \mathbf{e}_{j}\right)\left(\mathbf{T}^{\mathbf{i}+\mathbf{e}_{j}} \mathbf{e}_{j}\right)\right]
$$

where $C=\left(4 t_{0}^{2} / 50 U\right)\left[(9-2 \xi)-(2+\xi) \eta^{2}\right], \mathbf{e}_{1-3}$ are bcc unit vectors, and $\mathbf{e}_{0}$ is minus their sum (these vectors form a tetrahedral star around each site $\mathbf{i}$ ).

In the case $C<0$ the quadrupole moments order ferromagnetically (order I) along one of the trigonal axes of the crystal [Fig. 2(a)] with the interaction energy $E_{\text {vibr }}=(4 / 9) C$ per site. For positive $C$ the lowest configuration corresponds to antiferro ordering (II) of quadrupole moments along one of tetragonal axes [Fig. 2(b)] with $E_{\text {vibr }}=0$. We stress that these orders are stabilized already at the classical level. The phases shown in Fig. 2 have the vibronic states on sites: $a=\left(a_{x}+a_{y}+a_{z}\right) / \sqrt{3}$ for ferro and $a_{1,2}=\left(a_{x} \mp a_{y}\right) / \sqrt{2}$ for the two sublattices of antiferro order, where $a_{x}, a_{y}, a_{z}$ correspond to real combinations of the vibronic states (2). It results therefore that in both these orders the vibronic moments on sites are completely quenched (the same for orbital moments, of course). To investigate the excitation spectrum, we introduce on each site, following Ref. [15], two states orthogonal to the condensed state $a: \alpha=\left(a_{x}-a_{y}\right) / \sqrt{2}$,
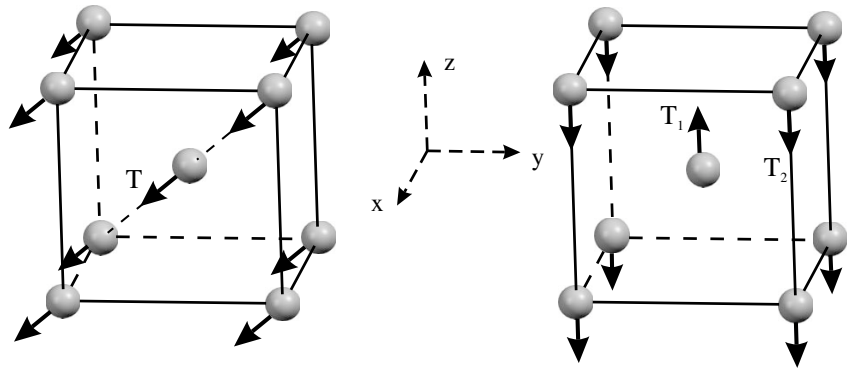

FIG. 2. Vibronic quadrupole ordered states: (a) ferro type (I), $T=(-2 / 3,-2 / 3,-2 / 3)$ and (b) antiffero type (II), $T_{1}=$ $(0,0,1), T_{2}=(0,0,-1)$. 


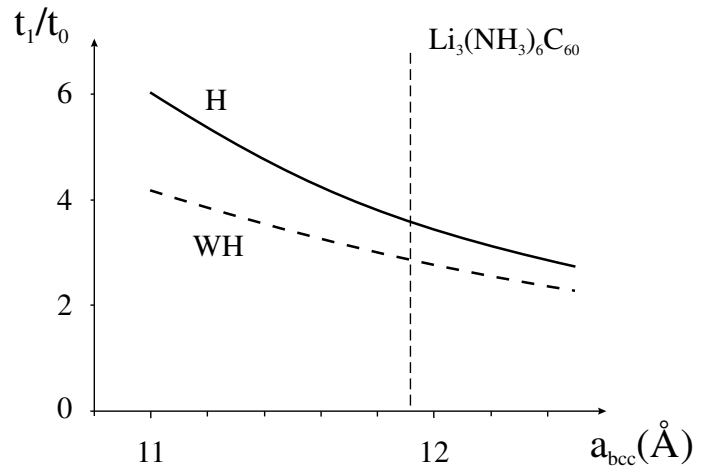

FIG. 3. Ratio of transfer parameters for LUMO orbitals of nearest neighbor fullerenes in a bcc lattice, calculated within Harrison and Wolfsberg-Helmholz approximations.

$\beta=\left(2 a_{z}-a_{x}-a_{y}\right) / \sqrt{6}$ for order $\mathrm{I}$ and $\alpha_{1,2}=$ $\left(a_{x} \pm a_{y}\right) / \sqrt{2}, \beta_{1,2}=a_{z}$ for two sublattices of order II.

The linearized Hamiltonian for the ferro phase has the form (in units of $8|C| / 9$ )

$$
\begin{aligned}
\mathcal{H}_{V W}^{\mathrm{I}}= & \sum_{\mathbf{i}}\left\{2\left(n_{\alpha}^{\mathbf{i}}+n_{\beta}^{\mathbf{i}}\right)-\frac{1}{9}\left[T_{\alpha}^{\mathbf{i}} T_{\alpha}^{\mathbf{i}+\mathbf{e}_{3}}\right.\right. \\
& +\left(\frac{1}{2} T_{\alpha}^{\mathbf{i}}-\frac{\sqrt{3}}{2} T_{\beta}^{\mathbf{i}}\right)\left(\frac{1}{2} T_{\alpha}^{\mathbf{i}+\mathbf{e}_{1}}-\frac{\sqrt{3}}{2} T_{\beta}^{\mathbf{i}+\mathbf{e}_{1}}\right) \\
& \left.\left.+\left(\frac{1}{2} T_{\alpha}^{\mathbf{i}}+\frac{\sqrt{3}}{2} T_{\beta}^{\mathbf{i}}\right)\left(\frac{1}{2} T_{\alpha}^{\mathbf{i}+\mathbf{e}_{2}}+\frac{\sqrt{3}}{2} T_{\beta}^{\mathbf{i}+\mathbf{e}_{2}}\right)\right]\right\},
\end{aligned}
$$

where $T_{\alpha} \approx-\left(\beta+\beta^{\dagger}\right)$ and $T_{\beta} \approx-\left(\alpha+\alpha^{\dagger}\right)$. This Hamiltonian describes two branches of optical magnons of vibronic origin with excitation energies $\omega_{ \pm}^{\mathbf{k}}=$ $\sqrt{1+\left(-C_{a} \pm \sqrt{C_{\theta}^{2}+C_{\epsilon}^{2}}\right) / 6}, \quad$ where $\quad C_{a}=\left(\cos k_{1}+\right.$ $\left.\cos k_{2}+\cos k_{3}\right) / 3, \quad C_{\theta}=\left(2 \cos k_{3}-\cos k_{1}-\cos k_{2}\right) / 6$, $C_{\epsilon}=\sqrt{3}\left(\cos k_{1}-\cos k_{2}\right) / 6$, and $k_{i}$ are projections of the momentum along the corresponding $\mathbf{e}_{i}$. The obtained spectrum of vibronic excitations has a relatively large gap and small dispersion, leading to a negligible number of bosons not in the condensate:

$$
\left\langle n_{\alpha}+n_{\beta}\right\rangle=\frac{1}{4 N} \sum_{\mathbf{k}}\left[\frac{\left(\omega_{+}^{\mathbf{k}}-1\right)^{2}}{\omega_{+}^{\mathbf{k}}}+\frac{\left(\omega_{-}^{\mathbf{k}}-1\right)^{2}}{\omega_{-}^{\mathbf{k}}}\right] \approx 0.001 .
$$

Accordingly the order parameter in this phase $\left\langle T_{z}\right\rangle=$ $\left\langle T_{x}\right\rangle=\left\langle T_{y}\right\rangle=-\left(2 / 3-\left\langle n_{\alpha}+n_{\beta}\right\rangle\right)$ is almost unreduced with respect to the classical equilibrium value [Fig. 2(a)].

The linearized Hamiltonian for the antiferro phase is of the form (in units of $8 C / 9$ )

$$
\mathcal{H}_{V W}^{\mathrm{II}}=\sum_{i}\left(2 n_{\alpha}^{i}+n_{\beta}^{i}\right),
$$

i.e., involving strictly local excitations. Thus the order parameters of both vibronic phases turn out to be almost unaffected by quantum fluctuations, which is the conse- quence of their classical origin. The parameter $C$ is much smaller than the constant part of $J_{i j}$ in (5), which means that the vibronic order sets in at a much lower temperature than the spin order.

Finally, we estimate the ratio between the two transfer parameters in orientationally ordered bcc fullerene crystals taking into account only the direct overlap of $t_{1 u}$ orbitals of nearest neighbor fullerenes. The overlap between carbon orbitals was calculated within Harrison (H) [17] and Wolfsberg-Helmholz (WH) approximations. Figure 3 shows that for the lattice size corresponding to $\mathrm{Li}_{3}\left(\mathrm{NH}_{3}\right)_{6} \mathrm{C}_{60}, \eta^{2} \approx 14(\mathrm{H})$ and $9(\mathrm{WH})$. These numbers are well above the critical value $(9-2 \xi) /(2+\xi) \approx 4.5$ $(\xi \approx 0)$ thus justifying the approximation made in (7). From these estimations we infer that $C<0$ implying the vibronic order of type I in the ground state of this fulleride.

In conclusion, the ground state and low-lying excitations in $\mathrm{Li}_{3}\left(\mathrm{NH}_{3}\right)_{6} \mathrm{C}_{60}$ are governed by spin-vibronic superexchange between neighbor fullerene sites. This interaction formally resembles the spin-orbital superexchange but involves a degenerate vibronic instead of orbital states on sites. The predictions of the spin-vibronic model are in line with available magnetic data for $\mathrm{Li}_{3}\left(\mathrm{NH}_{3}\right)_{6} \mathrm{C}_{60}$. We suggest that a quadrupole vibronic order develops at a temperature much lower than the temperature of spin ordering transition, which could be tested in future experiments.

I thank A. M. Oleś for useful discussions. This work has been supported by the Belgian Science Foundation and Flemish Government under Concerted Action Scheme.

[1] O. Gunnarsson, Rev. Mod. Phys. 69, 575 (1997).

[2] M. J. Rosseinsky, Chem. Mater. 10, 2665 (1998).

[3] M. Imada, A. Fujimori, and Y. Tokura, Rev. Mod. Phys. 70, 1039 (1998).

[4] Y. Tokura and N. Nagaosa, Science 288, 462 (2000).

[5] P. Durand et al., Nat. Mater. 2, 605 (2003).

[6] Y. Iwasa et al., Phys. Rev. B 53, R8836 (1996).

[7] K. I. Kugel' and D. I. Khomskii, Sov. Phys. Usp. 25, 231 (1982).

[8] A. M. Oleś, M. Cuoco, and N. B. Perkins, in Lectures of the Physics of Highly Correlated Electron Systems IV, edited by F. Mancini, AIP Conf. Proc. No. 527 (AIP, New York, 2000).

[9] M. C. M. O’Brien, Phys. Rev. 187, 407 (1969).

[10] I. B. Bersuker and V.Z. Polinger, Vibronic Interactions in Molecules and Crystals (Springer, Berlin, 1989).

[11] A. Auerbach, N. Manini, and E. Tosatti, Phys. Rev. B 49, 12998 (1994); N. Manini, E. Tosatti, and A. Auerbach, ibid. 49, 13008 (1994).

[12] M. C. M. O’Brien, Phys. Rev. B 53, 3775 (1996).

[13] P. W. Anderson, Phys. Rev. 115, 2 (1959).

[14] L. F. Chibotaru (unpublished).

[15] G. Khaliullin and S. Okamoto, Phys. Rev. Lett. 89, 167201 (2002); Phys. Rev. B 68, 205109 (2003).

[16] G. Khaliullin and S. Maekawa, Phys. Rev. Lett. 85, 3950 (2000).

[17] O. Gunnarsson et al., Phys. Rev. B 57, 2159 (1998). 